\title{
Best of The Lighter Side (1992-2009)
}

Tobacco control is not a particularly cheery endeavour. Public health advocates are lambasted as Nanny Staters and Health Nazis. And we are, after all, discussing an epidemic that kills 5 million people a year now and will take a billion lives prematurely in this century if we don't rein in the tobacco industry sometime soon. Still, there is wry humour to be found amid the grimness and some cartoonists have artfully illustrated the inherent contradictions of an industry and its products that are, at heart, deadly poisons wrapped like chocolate treats.

No image has ever more succinctly illustrated the 'logic' of tobacco's defenders than Wiley's Non Sequitur 'Physics as understood by restaurateurs' cartoon. And Tom Toles of the Buffalo News summarised perfectly Tobacco Inc's problematic case back in 1994, thus: "We have developed a new cigarette. With almost no annoying smoke, not that smoke is annoying. And $90 \%$ less of the cancer-causing tars, not that the tars in our other cigarettes cause cancer. But it still has $100 \%$ of the nicotine, not that nicotine is the addictive part."

Here are those and a handful more of the best cartoons to have appeared in these pages over the past 20 years.

\section{Curated By Norbert Hirschhorn and Stan Shatenstein}

\section{ADVERTISING}

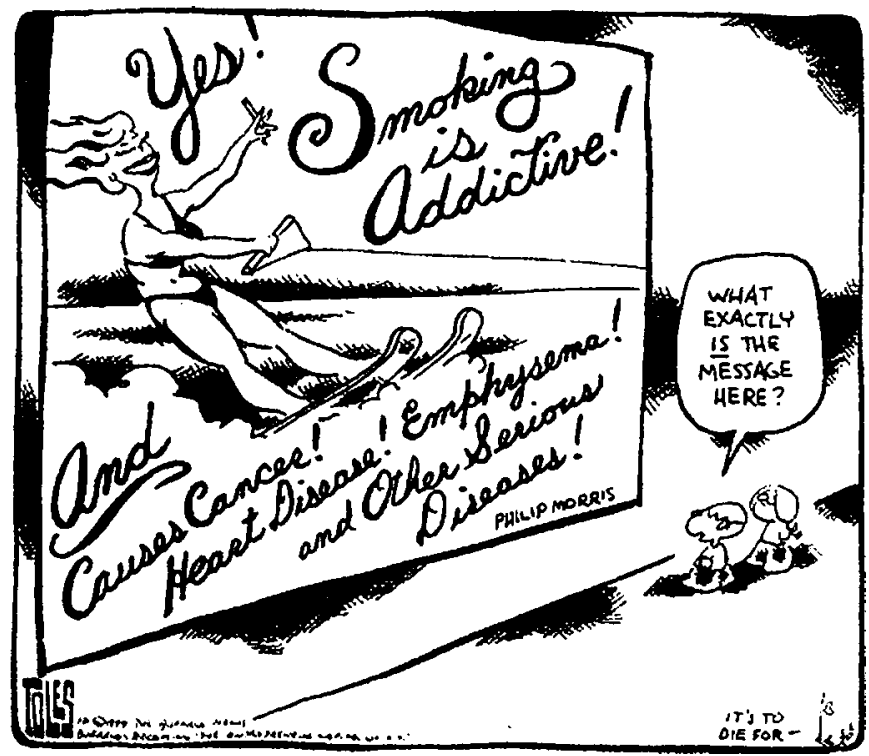

1999:8:4:386.

http://tc.bmjjournals.com/cgi/content/full/8/4/378 


\section{HEALTH AND ADDICTION}
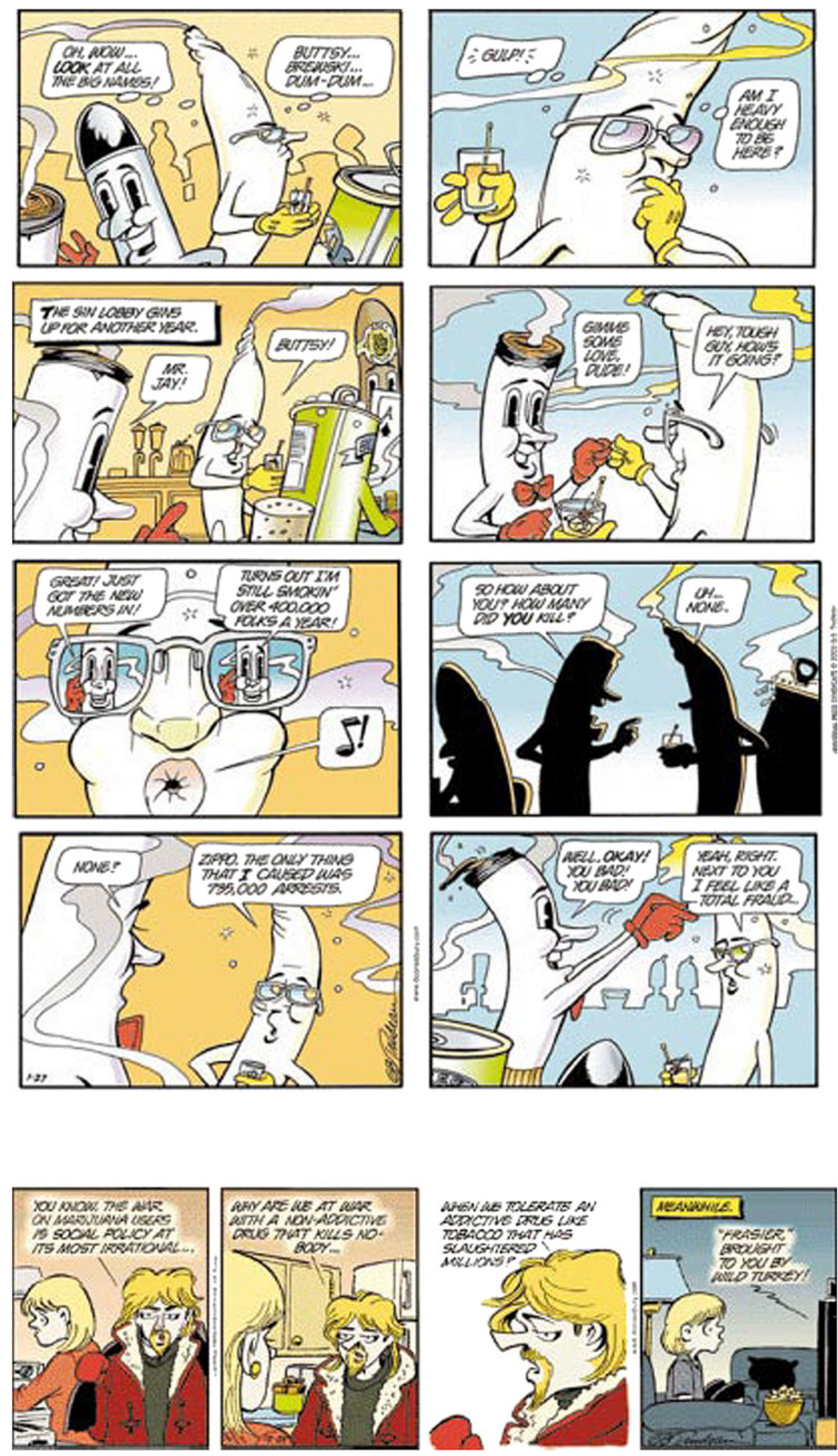

2002;11:2:157-8.

http://tc.bmjjournals.com/cgi/content/full/11/2/157 


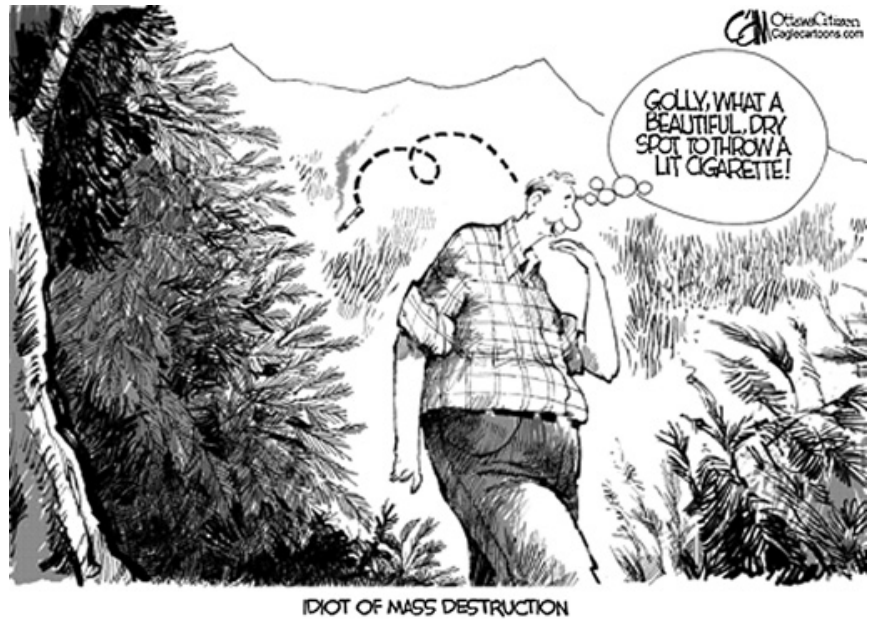

2004:13:4:408.

http://tobaccocontrol.bmj.com/content/13/4.toc\#Miscellanea

\section{SECONDHAND SMOKE AND SMOKING BANS}

NON SEQUITUR

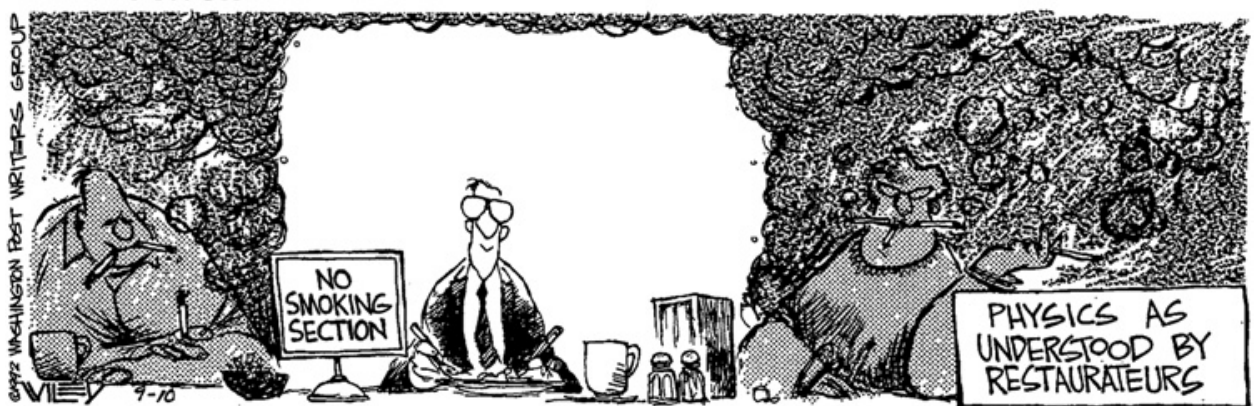

Copyright 1992, Washington Post Writers Group. Reprinted with permission

1993:2:3:242-3

http://tc.bmjjournals.com/cgi/reprint/2/3/242.pdf

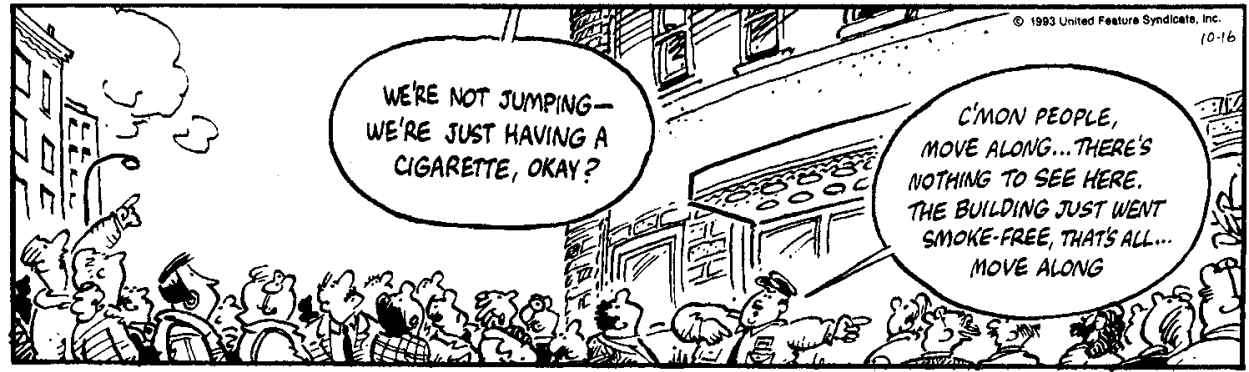

$B E T T Y$, reprinted with permission of UFS, Inc

1994:3:4:370

http://tc.bmijournals.com/cgi/reprint/3/4/370.pdf 


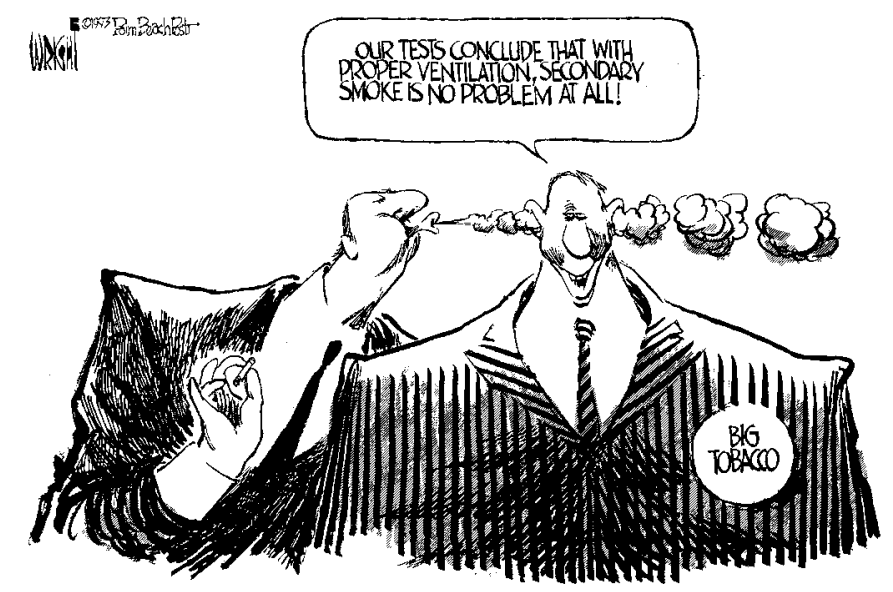

Reprinted with permission of Don Wright, The Palm Beach Post.

1994:3:1:76-7.

http://tc.bmjjournals.com/cgi/reprint/3/1/76.pdf

THE NEFARIOUS INDUSTRY AND TOBACCO CONTROL

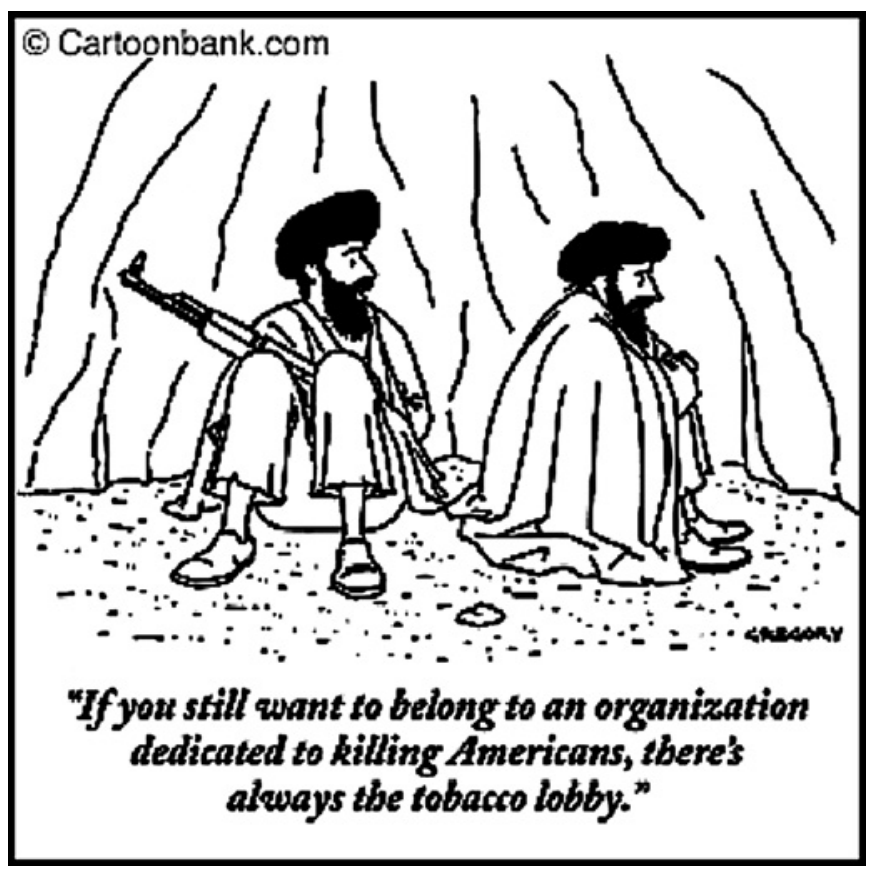

2002:11:3:245.

http://tc.bmjjournals.com/cgi/content/full/11/3/245 

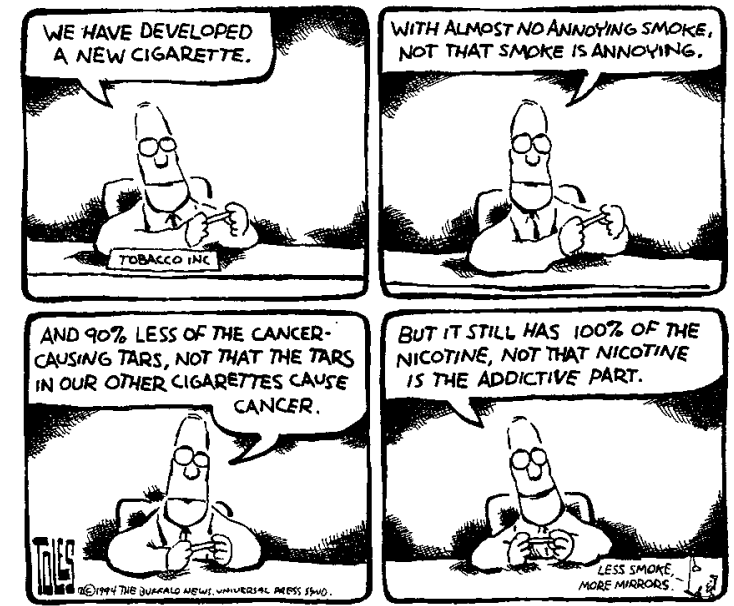
NICOTINE NOT THAT NICOTINE IS TUE ADOICTIVE PART.

By TOLES (C) 1994, The Buffalo News, reprinted with permission of UNITED PRESS SYNDICATE. All rights reserved.

1995:4:3:280-1.

http://tc.bmjjournals.com/cgi/reprint/4/3/280.pdf

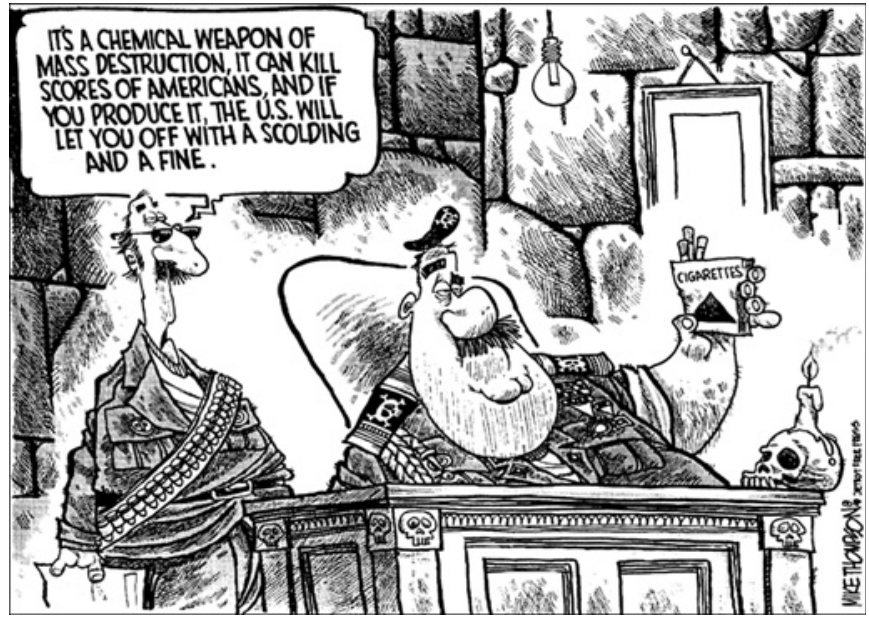

1999:8:1:92.

http://tc.bmjjournals.com/cgi/content/full/8/1/92/
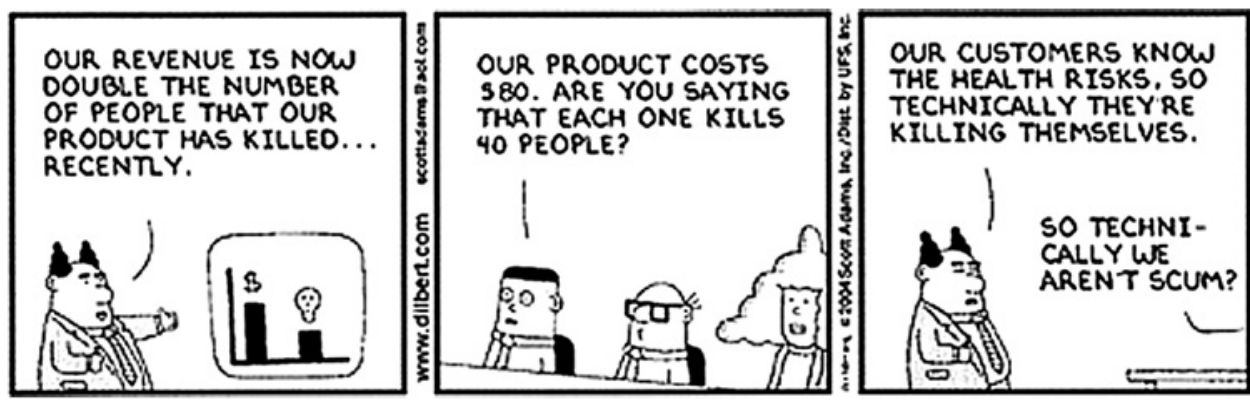

2005:14:2:113.

http://tobaccocontrol.bmj.com/content/14/2.toc\#Thelighterside 


\section{QUITTING}

CHAOS by Brian Shuster

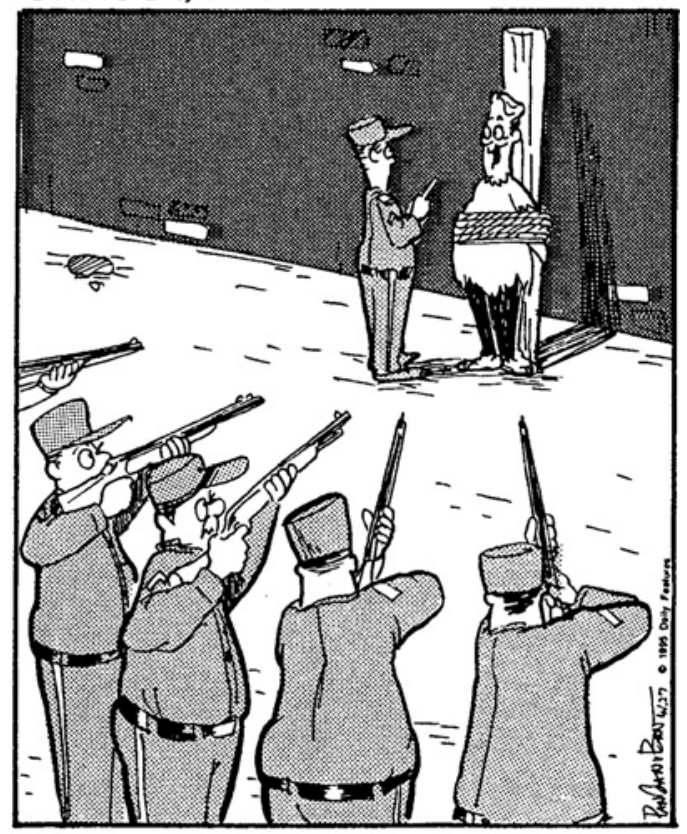

"No thanks, I'm wearing the Patch."

CHAOS, by Brian Shuster. Reprinted by permission of Daily Features.

1995:4:4:391-2.

http://tc.bmjjournals.com/cgi/reprint/4/4/391.pdf

\section{MARKETING TO KIDS}

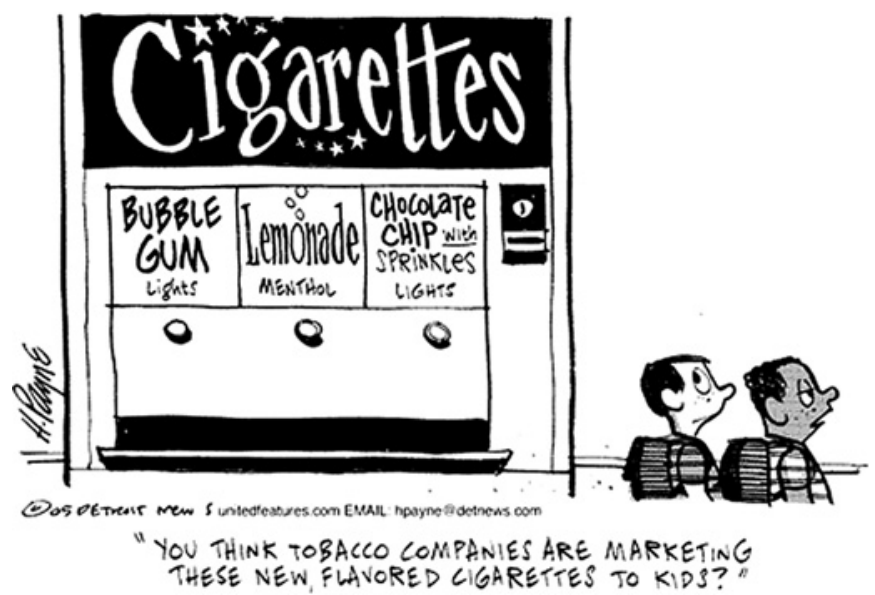

2006:15:1:63.

http://tobaccocontrol.bmj.com/content/15/1.toc\#Miscellanea

Competing interests None.

Provenance and peer review Commissioned; not internally peer reviewed.

Accepted 5 January 2012

Tobacco Control 2012;21:202-207. doi:10.1136/tobaccocontrol-2012-050425 